\title{
The Relationship Between Triglycerides, High- Density Lipoprotein, Triglyceride/High-Density Lipoprotein, and Hospital Death in Patients with Sepsis: A Retrospective Cohort Study
}

\section{Rongpeng Gong ( $\nabla 728955 @ q q . c o m$ )}

Qinghai Medical College: Qinghai University Medical College https://orcid.org/0000-0003-1117-0956

Yu Tian

Anyue Country pepole's hospitak

Tianyang Hu

Chongqing Medical University

\section{Zixin Xu}

Shaanxi University of Chinese Medicine

\section{Xiao Deng}

Caojie community Health Services center

\section{Xiaofang Shen}

Aerospace Central Hospital: Central Hospital of China Aerospace Corporation

\section{Xiaoxin Wei}

Qinghai Medical College: Qinghai University Medical College

\section{Research}

Keywords: triglycerides, High-density lipoprotein, Cox regression, sepsis, hospitalization death

Posted Date: August 13th, 2021

DOI: https://doi.org/10.21203/rs.3.rs-793028/v1

License: (9) (i) This work is licensed under a Creative Commons Attribution 4.0 International License. Read Full License 


\section{Abstract}

Background:

Sepsis remains the leading cause of death in the ICU. There is still controversy about the association between TG, HDL and TG/HDL and sepsis. Our aim was to explore the relationship between TG, HDL, TG/HDL and the hospitalized mortality of sepsis by using the MIMIC-IV database.

Methods:

Selected from patients admitted to MIMIC-IV database (2008-2019). A total of 1032 eligible participants were included. Multivariate Cox regression model was used to analyze the correlation between TG, HDL, TG/HDL and sepsis hospitalization death after excluding potential confounding factors, and the smooth curve fitting graph and survival curve were drawn. Subgroup analysis was performed to test the results.

Results

There was a negative correlation between TG , TG/HDL and the poor prognosis of sepsis, while HDL was positively correlated with the poor prognosis of sepsis.

\section{Introduction}

Sepsis is a life-threatening organ dysfunction syndrome with high mortality caused by the dysregulated host response to infection [1]. A retrospective study by Kristina E Rudd et al. showed that there were 48.9 million sepsis cases recorded globally in 2017, and 11 million sepsis-related deaths, accounting for $19.7 \%$ of the total[2]. In 2020, C Fleischmann-Struzek et al.[3] conducted a meta-analysis and found that the incidence of sepsis in inpatients was 18.9 individuals per million person-years, and the mortality rate was $26.7 \%$; the incidence of sepsis in intensive care patients was 58 individuals per million person-years, and the mortality rate was as high as $41.9 \%$ in intensive care units (ICU). Not only do patients with sepsis have a high mortality rate, but the survivors also have a high disability rate, resulting in functional disability, cognitive impairment, and the great challenge of a decline in the quality of life [4]. In addition, the economic losses caused by sepsis to society should not be underestimated. In 2018 , the direct economic losses caused by sepsis in the United States amounted to 28 billion dollars[5], and survivors also face the decline in productivity caused by disability, and bear the economic burden of direct medical costs, nursing costs, and increased demand for informal care[4]. The high morbidity, high mortality, and high economic burden of sepsis have caused great troubles to human health and life, and made it a public health issue of global concern. Effectively preventing and treating sepsis and improving its prognosis have become the main challenges faced by medical staff, especially in ICU. Thus, early prediction and intervention have become the focus of sepsis research.

Clinically, triglycerides (TG) and high-density lipoprotein (HDL) have become routine laboratory examination items for most hospitalized patients. In addition, TG and HDL have been used to predict the 
occurrence and development of various diseases such as chronic kidney disease, diabetes, coronary heart disease [6-12], and have achieved good predictive effects. Meanwhile, studies have reported that the effectiveness of predicting the occurrence and development of the disease by the ratio of TG to HDL (TG/HDL) is more accurate than the effectiveness of a single TG or HDL [13-17]. Morin et al. believed that TG and HDL play a vital role in the pathophysiological process of sepsis, especially the antiapoptotic effect and endothelial protection of HDL could limit the progression of sepsis and reduce its severity [18]. However, the correlation between TG, HDL and TG/HDL levels and the mortality of sepsis has not been fully investigated. In this study, we aim to use the public medical database (Medical Information Mart for Intensive Care-IV, MIMIC-IV) to explore the correlation between the three factors (TG, $\mathrm{HDL}$, and TG/HDL) and in-hospital mortality of intensive care patients with sepsis, and to find the underlying mechanisms.

\section{Methods}

\subsection{Database}

The MIMIC-IV database contains data of patients admitted to the critical care units of the Beth Israel Deaconess Medical Center from 2008 to 2019, and has been greatly improved on the basis of the MIMICIII database [19]. Any researcher who follows the usage requirements can access the database. The author Tianyang Hu passed the "Protecting Human Research Participants" exam (Record ID: 37474354) on the National Institutes of Health $(\mathrm{NIH})$ website and was allowed to extract data. The information about patients in the database is anonymous, and there is no need to obtain their informed consent.

\subsection{Study populations}

The inclusion criteria of this study were: 1) Patients diagnosed with sepsis during admission to the ICU (SOFA score $\geq 2$ and suspected of infection); 2) Patients older than 18 years old; 3 ) TG and HDL levels were measured within 24 hours after admission. Since the same patient may be admitted to the ICU multiple times, we only select the data of the first ICU admission for each patient who meets these criteria.

\subsection{Data extraction}

The data was extracted from the MIMIC-IV database (version 1.0). Use Navicat Premium 15.0 software to extract the following variables of the included patients: age, gender, ethnicity, Sequential Organ Failure Assessment (SOFA) score, Charlson comorbidity index, hemoglobin (HB), platelets (PLT), white blood cells (WBC), red blood cells (RBC), albumin (ALB), blood urea nitrogen (BUN), creatinine (CRE), anion gap (AG), alanine aminotransferase (ALT), Aspartate aminotransferase (AST), total bilirubin (TBIL), glucose (GLU), heart rate, systolic blood pressure (SBP), diastolic blood pressure (DBP), respiratory rate, body temperature, length of hospital stay, length of ICU stay, hospital expire flag (indicating whether the patient died during hospitalization), date of death, etc. If a variable was measured multiple times within 24 hours after admission, the average value was taken. 


\subsection{Statistical analysis}

All statistical analysis was performed with $\mathrm{R}$ software (version 4.0.2). Continuous variables were expressed in detailed sample descriptions, with an average confidence interval (Cl) of 95\%. Categorical variables were expressed as counts and weighted percentages[20]. The Student's t test or Mann-Whitney $U$ test based on normality of distribution was used to compare continuous variables between groups, and the Fisher exact test was used to compare categorical variables between groups. We used multiple imputation method to fill in the missing data to make the data complete. To observe whether the generated complete data was significantly different from the original data, we conducted a sensitivity analysis. The results showed that the complete data after interpolation was not much different from the original data. Therefore, we used the imputed data for multivariate analysis, which also conformed to the Rubin rule.

There may be potential confounding factors among TG, HDL, TG/HDL and in-hospital mortality of sepsis. Based on previous studies, international standards and clinical experience, age, gender, ethnicity, SOFA score, WBC, AG, ALT, AST, ALB, TBIL, CRE, BUN, SBP, DBP, and GLU were selected as the covariates after comprehensive consideration. We designed three steps for the statistical analysis:

1. Step 1: Weighted univariate and multivariate Cox regression models. We built four models: Model 1, without adjusting any covariates; Model 2, adjusting for gender, age, and ethnicity; Model 3, adding SOFA scores, AG and WBC on the basis of Model 2; Model 4, adding ALT, AST, ALB, TBIL, CRE, BUN, SBP, DBP on the basis of model 3;

2. Step 2: To explore the correlation between TG, HDL, TG/HDL and in-hospital mortality of intensive care patients with sepsis, smoothing splines were performed;

3. Step 3: Subgroup analysis was performed with a weighted stratified Cox regression model to ensure the stability of the results. Continuous variables were first converted to categorical variables based on clinical cut-off values or percentiles, and then the interaction test was performed. The effect modification test was carried out by the interaction terms between the subgroup indicators, and then the likelihood ratio test was performed.

\section{Result}

\subsection{Characteristics of the study population}

In this study, the names of septic patients with TG or HDL data and consistent with the definition of Sepsis-3 were obtained from MIMIC-IV (2008-2019). Children ( $<18$ years old) and participants with single TG or HDL were excluded. In the end, a total of 1032 people were included in the study (Fig. 1), among which 196 participants died and 836 did not die. The death rate of males (620 cases, 61.2\%) was higher than that of females (76 cases, $38.8 \%$ ). The age of dead participants (69.4 15.8) was slightly higher than that of non-dead participants (67.4 15.9). We observed that the scores of BUN, CRE, AST, ALT, TBIL, WBC and SOFA of non-dead participants were lower. In contrast, SBP, HBP, MBP, TG, and length of 
hospital stay were lower for participants with an outcome of death. There was no significant difference between participants who died and those who did not die in terms of whether they had liver disease, diabetes, hypertension and other indicators. (Table 1) 
Table 1

Baseline characteristics of the study participants

Table 1. Baseline characteristics of the study participants

\begin{tabular}{|c|c|c|c|c|}
\hline Variables & Total $(n=1032)$ & $\begin{array}{l}\text { non-death }(n= \\
836)\end{array}$ & death $(n=196)$ & $\begin{array}{l}\mathrm{P}- \\
\text { value }\end{array}$ \\
\hline Age, year, Mean \pm SD & $67.8 \pm 15.9$ & $67.4 \pm 15.9$ & $69.4 \pm 15.8$ & 0.113 \\
\hline Gender, n (\%) & & & & 0.088 \\
\hline male & $573(55.5)$ & $453(54.2)$ & $120(61.2)$ & \\
\hline female & $459(44.5)$ & $383(45.8)$ & $76(38.8)$ & \\
\hline Ethnicity, n (\%) & & & & $<0.001$ \\
\hline white & $608(58.9)$ & $516(61.7)$ & $92(46.9)$ & \\
\hline black & $92(8.9)$ & $76(9.1)$ & $16(8.2)$ & \\
\hline others & $332(32.2)$ & $244(29.2)$ & $88(44.9)$ & \\
\hline Height, cm, Mean \pm SD & $168.8 \pm 11.7$ & $168.7 \pm 11.7$ & $169.6 \pm 11.7$ & 0.325 \\
\hline Weight, kg, Mean \pm SD & $83.3 \pm 23.9$ & $82.8 \pm 24.2$ & $85.3 \pm 22.8$ & 0.192 \\
\hline $\begin{array}{l}\text { LOS hospital, day, Mean } \pm \\
\text { SD }\end{array}$ & $13.5 \pm 13.1$ & $14.5 \pm 13.6$ & $9.3 \pm 9.7$ & $<0.001$ \\
\hline SOFA score, Mean \pm SD & $3.4 \pm 1.9$ & $3.2 \pm 1.8$ & $3.8 \pm 2.3$ & $<0.001$ \\
\hline $\mathrm{SBP}, \mathrm{mmHg}$, Mean $\pm \mathrm{SD}$ & $125.1 \pm 19.2$ & $125.6 \pm 18.4$ & $123.0 \pm 22.1$ & 0.09 \\
\hline $\mathrm{DBP}, \mathrm{mmHg}$, Mean $\pm \mathrm{SD}$ & $66.4 \pm 11.9$ & $66.7 \pm 11.8$ & $65.1 \pm 12.4$ & 0.099 \\
\hline MBP, mmHg, Mean \pm SD & $83.0 \pm 12.0$ & $83.3 \pm 11.8$ & $81.5 \pm 12.6$ & 0.057 \\
\hline WBC, 109/L, Median (IQR) & $11.2(8.7,14.6)$ & $10.9(8.5,14.3)$ & $12.7(9.7,15.8)$ & $<0.001$ \\
\hline Liver disease, n (\%) & & & & 0.986 \\
\hline no & $871(84.4)$ & 705 (84.3) & $166(84.7)$ & \\
\hline yes & $161(15.6)$ & $131(15.7)$ & $30(15.3)$ & \\
\hline Diabetes, n (\%) & & & & 0.955 \\
\hline no & 717 (69.5) & $580(69.4)$ & $137(69.9)$ & \\
\hline yes & 315 (30.5) & $256(30.6)$ & $59(30.1)$ & \\
\hline Hypertension, n (\%) & & & & 0.781 \\
\hline no & $541(52.4)$ & $436(52.2)$ & $105(53.6)$ & \\
\hline yes & $491(47.6)$ & $400(47.8)$ & $91(46.4)$ & \\
\hline
\end{tabular}




\begin{tabular}{|c|c|c|c|c|}
\hline ALB, g/dL, Median (IQR) & $3.5(3.1,3.9)$ & $3.5(3.1,3.9)$ & $3.5(2.9,3.8)$ & 0.095 \\
\hline BUN, mmol/L, Median (IQR) & $19.8(14.4,30.5)$ & $19.0(13.5,29.5)$ & $24.0(16.4,36.5)$ & $<0.001$ \\
\hline CRE, ng/dL, Median (IQR) & $1.1(0.8,1.6)$ & $1.0(0.8,1.4)$ & $1.2(0.9,2.1)$ & $<0.001$ \\
\hline ALT, U/L, Median (IQR) & $28.0(17.0,81.0)$ & $27.0(16.0,75.0)$ & $\begin{array}{l}33.0(18.9 \\
100.4)\end{array}$ & 0.026 \\
\hline AST, U/L, Median (IQR) & $\begin{array}{l}43.0(24.0 \\
140.5)\end{array}$ & $41.0(23.5,132.6)$ & $\begin{array}{l}50.2(29.8 \\
183.4)\end{array}$ & 0.002 \\
\hline TBIL, mg/dL, Median (IQR) & $0.6(0.4,1.1)$ & $0.6(0.4,1.0)$ & $0.7(0.5,1.1)$ & 0.036 \\
\hline TG, mmol/L, Median (IQR) & $1.2(0.8,1.7)$ & $1.2(0.8,1.7)$ & $1.1(0.9,1.6)$ & 0.595 \\
\hline HDL, mmol/L, Median (IQR) & $1.1(0.8,1.4)$ & $1.1(0.8,1.4)$ & $1.1(0.8,1.3)$ & 0.283 \\
\hline TG/HDL, Median (IQR) & $1.1(0.7,2.0)$ & $1.1(0.7,2.0)$ & $1.1(0.7,1.9)$ & 0.678 \\
\hline \multicolumn{5}{|c|}{$\begin{array}{l}\text { SD = Standard Deviation; LOS = Length of Stay; SOFA = Sequential Organ Failure Assessment; SBP = } \\
\text { Systolic Blood Pressure; DBP = Diastolic Blood Pressure; MBP = Mean Blood Pressure; WBC = White } \\
\text { Blood Cell; IQR = Interquartile Range; ALB = Albumin; BUN = Blood Urea Nitrogen; CRE = Creatinine; ALT } \\
\text { = Alanine aminotransferase; AST = Aspartate aminotransferase; TBIL = Total Bilirubin; TG = } \\
\text { Triglycerides; HDL = High-Density Lipoprotein. }\end{array}$} \\
\hline
\end{tabular}

\subsection{Univariate analyses}

This study analyzed the correlation between sex, age, race, liver function (ALT, AST, TBIL), blood lipids (HDL, TG, TG/HDL), renal function (BUN, CRE), SOFA score and hospital mortality of sepsis. In Table 2, women are less likely to die than men, with an effect size and $95 \%$ confidence interval of $0.82(0.61,1.09)$. The values of ALB, SBP, DBP, RBC, TG and HDL were negatively correlated with the hospital mortality of sepsis, while the SOFA score and AG, BUN, WBC, CRE were positively correlated with the hospital mortality of sepsis. (Table 2) 
Table 2

Univariate analysis for Sepsis in-hospital mortality.

\begin{tabular}{lll}
\multicolumn{2}{l}{ Table 2. Univariate analysis for Sepsis in-hospital mortality } \\
\hline Variable & HR( $(95 \% \mathrm{Cl})$ & P-value \\
\hline Age & $1.01(1,1.02)$ & 0.009
\end{tabular}

Ethnicity

white 1

$\begin{array}{lll}\text { black } & 1.03(0.61,1.76) & 0.903 \\ \text { others } & 1.56(1.17,2.1) & 0.003\end{array}$

Gender

$\begin{array}{lll}\text { male } & 0.82(0.61,1.09) & 0.175\end{array}$

$\begin{array}{lll}\text { female } & 0.82(0.61,1.09) & 0.175\end{array}$

$\begin{array}{lll}\text { SOFA score } & 1.07(1.01,1.13) & 0.021\end{array}$

$\begin{array}{lll}\text { ALB } & 0.77(0.61,0.98) & 0.032\end{array}$

AG $\quad 1.09(1.05,1.12) \quad<0.001$

BUN $1.01(1,1.02)<0.001$

$\begin{array}{lll}\text { CRE } & 0.035\end{array}$

$\begin{array}{lll}\text { ALT } & 1(0.9998,1.0002) & 0.837\end{array}$

$\begin{array}{lll}\text { AST } & 1(0.9999,1.0001) & 0.947\end{array}$

$\begin{array}{lll}\text { TBIL } & 1.01(0.99,1.04) & 0.326\end{array}$

$\begin{array}{lll}\text { Heart rate } & 1.0044(0.9962,1.0128) & 0.293\end{array}$

$\begin{array}{lll}\text { SBP } & 0.99(0.98,1) & 0.011\end{array}$

$\begin{array}{lll}\text { DBP } & 0.98(0.97,1) & 0.008\end{array}$

$\begin{array}{lll}\text { MBP } & 0.98(0.97,0.99) & <.001\end{array}$

$\begin{array}{lll}\text { WBC } & 1.01(1,1.02) & 0.004\end{array}$

$\begin{array}{lll}\text { RBC } & 0.95(0.78,1.14) & 0.557\end{array}$

$\begin{array}{lll}\text { GLU } & 1(1,1) & 0.047\end{array}$

$\begin{array}{lll}\text { TG } & 0.95(0.87,1.03) & 0.215\end{array}$

$\begin{array}{lll}\text { HDL } & 0.86(0.64,1.15) & 0.309\end{array}$

$\begin{array}{lll}\text { TG/HDL } & 0.98(0.95,1.02) & 0.293\end{array}$ 


\section{Table 2. Univariate analysis for Sepsis in-hospital mortality}

HR = Hazard Ratio; SOFA = Sequential Organ Failure Assessment; ALB = Albumin; AG = Anion Gap; BUN = Blood Urea Nitrogen; CRE = Creatinine; ALT = Alanine aminotransferase; AST = Aspartate aminotransferase; TBIL = Total Bilirubin; SBP = Systolic Blood Pressure; DBP = Diastolic Blood Pressure; MBP = Mean Blood Pressure; WBC = White Blood Cell; RBC = Red Blood Cell; GLU = Glucose; TG = Triglycerides; HDL = High-Density Lipoprotein.

\subsection{Multivariate analysis}

\subsubsection{Relationship between TG and hospital mortality in sepsis}

We established four cox regression models to explore the relationship between TG and hospital mortality in sepsis. Model 1, without adjusting any covariables; Model 2 adjusted only for gender, age, and race; In Model 3, SOFA score, anion gap and WBC were added on the basis of Model 2. Model 4, on the basis of model 3 increased ALT, AST, propagated, TBIL, CRE, BUN, SBP, DBP. The model-based effect size can be interpreted as a proportional reduction in the risk of death in sepsis patients for each 1-unit increase in TG. In the unadjusted model, for example, the HR was 0.95 (0.77-1.03), meaning that a one-unit increase in TG was associated with a $5 \%$ reduction in sepsis risk. In the model adjusted only based on sociodemographic data (model 2), for each additional unit of TG, the risk of sepsis decreased by $7 \%$ (0.93 $(0.85,1.03))$. In the further adjusted model (model 3), for each additional unit of TG, the risk of sepsis decreased by $8 \%(0.92(0.90,0.94))$. In the fully adjusted model (model 4$)$, each additional unit of vitamin D3 reduced the risk of sepsis by $9 \%(0.91(0.83,1.01))$. For sensitivity analysis, we convert the value of TG from continuous variables to classified variables. The P value of the changing trend of TG in model 4 is consistent with that of continuous variables. In addition, we also found that the effects of different TG levels showed an isometric trend. (Table 3)

Table 3

The association between TG, HDL and TG/HDL for Sepsis in-hospital mortality in a multiple model

\begin{tabular}{|c|c|c|c|c|c|c|c|c|}
\hline \multirow[t]{2}{*}{ Variable } & \multicolumn{2}{|l|}{ Model 1} & \multicolumn{2}{|l|}{ Model 2} & \multicolumn{2}{|l|}{ Model 3} & \multicolumn{2}{|l|}{ Model 4} \\
\hline & $\mathrm{HR}(95 \% \mathrm{Cl})$ & $\begin{array}{l}P \text { - } \\
\text { value }\end{array}$ & $\mathrm{HR}(95 \% \mathrm{Cl})$ & $\begin{array}{l}\text { P. } \\
\text { value }\end{array}$ & $\mathrm{HR}(95 \% \mathrm{Cl})$ & $\begin{array}{l}P \text { - } \\
\text { value }\end{array}$ & $\mathrm{HR}(95 \% \mathrm{Cl})$ & $\begin{array}{l}\mathrm{P} \text { - } \\
\text { value }\end{array}$ \\
\hline TG/HDL & $\begin{array}{l}0.98(0.95 \\
\sim 1.02)\end{array}$ & 0.293 & $\begin{array}{l}0.97(0.93 \\
\sim 1.02)\end{array}$ & 0.209 & $\begin{array}{l}0.96(0.92 \\
\sim 1.01)\end{array}$ & 0.093 & $\begin{array}{l}0.95(0.91 \\
\sim 1)\end{array}$ & 0.036 \\
\hline $\mathrm{TG}$ & $\begin{array}{l}0.95(0.87 \\
\sim 1.03)\end{array}$ & 0.215 & $\begin{array}{l}0.93(0.85 \\
\sim 1.03)\end{array}$ & 0.174 & $\begin{array}{l}0.91(0.83 \\
\sim 1.01)\end{array}$ & 0.086 & $\begin{array}{l}0.90(0.80 \\
\sim 1.0)\end{array}$ & 0.049 \\
\hline HDL & $\begin{array}{l}0.86(0.64 \\
\sim 1.15)\end{array}$ & 0.309 & $\begin{array}{l}0.78(0.57 \\
\sim 1.06)\end{array}$ & 0.116 & $\begin{array}{l}1.07(0.74 \\
\sim 1.54)\end{array}$ & 0.727 & $\begin{array}{l}1.14(0.8 \\
\sim 1.63)\end{array}$ & 0.469 \\
\hline \multicolumn{9}{|c|}{$\begin{array}{l}\text { Model 1, without adjusting any covariates; Model 2, adjusting for gender, age, and ethnicity; Model } 3 \text {, } \\
\text { adding SOFA scores, AG and WBC on the basis of Model 2; Model 4, adding ALT, AST, ALB, TBIL, CRE, } \\
\text { BUN, SBP, DBP on the basis of model 3; }\end{array}$} \\
\hline
\end{tabular}




\subsubsection{Relationship between HDL and hospital mortality in sepsis}

We established four cox regression models to explore the relationship between $\mathrm{HDL}$ and hospital mortality in sepsis. Model 1, without adjusting any covariables; Model 2 adjusted only for gender, age, and race; In Model 3, SOFA score, anion gap and WBC were added on the basis of Model 2. Model 4, on the basis of model 3 increased ALT, AST, propagated, TBIL, CRE, BUN, SBP, DBP. The model-based effect size can be interpreted as a proportional reduction in the risk of death in sepsis patients for each 1-unit increase in HDL. For example, the effect value of the unadjusted model is HR 0.86 (0.64-1.15), which means that each additional unit of HDL reduces the risk of death in patients with sepsis by $14 \%$. In the model adjusted only for sociodemographic data (model 2), each additional unit of HDL reduced the risk of in-hospital mortality in patients with sepsis by $22 \%(0.78(0.57-1.06))$. In the further adjusted model (model 3), each additional unit of HDL increased the risk of hospital mortality in patients with sepsis by $7 \%(1.07(0.74-1.54))$. In the fully adjusted model (model 4), each additional unit of HDL increased the risk of in-hospital mortality in patients with sepsis by $14 \%(1.14(0.8-1.63))$. For sensitivity analysis, we convert the value of HDL from continuous variables to classified variables. The $P$ value of the changing trend of $\mathrm{HDL}$ in model 4 is consistent with that of continuous variables. In addition, we also found that the effects of different HDL levels showed an isometric trend. (Table 3)

\subsubsection{Relationship between TG/HDL and hospital mortality in sepsis}

We established four cox regression models to explore the relationship between TG/HDL and hospital mortality in sepsis. Model 1, without adjusting any covariables; Model 2 adjusted only for gender, age, and race; In Model 3, SOFA score, anion gap and WBC were added on the basis of Model 2. Model 4, on the basis of model 3 increased ALT, AST, propagated, TBIL, CRE, BUN, SBP, DBP. The model-based effect size can be interpreted as a proportional reduction in the risk of death in sepsis patients for each 1-unit increase in TG/HDL. For example, the effect value of the unadjusted model is HR 0.98 (0.95 to 1.02), which means that each additional unit of TG/HDL reduces the risk of death in patients with sepsis by $2 \%$. In the model adjusted only based on sociodemographic data (model 2), for each unit increase in TG/HDL, the risk of hospital mortality in patients with sepsis decreased by $3 \%$ (0.97 (0.93 to 1.02)). In a further adjusted model (model 3 ), each additional unit of TG/HDL reduced the risk of in-hospital mortality in septic patients by $4 \%(0.96(0.92-1.01))$. In the fully adjusted model (model 4$)$, for each additional unit of $\mathrm{TG} / \mathrm{HDL}$, the risk of in-hospital mortality in patients with sepsis decreased by $5 \%(0.95$ (0.91-1)). For sensitivity analysis, we convert the value of TG/HDL from continuous variables to classified variables. The $P$ value of the changing trend of TG/HDL in model 4 is consistent with that of continuous variables. In addition, we also found that the effects of different TG/HDL levels showed an isometric trend. (Table 3)

\subsection{Smooth curve fitting and survival analysis}


In this study, we analyzed the linear relationship between TG, HDL, TG/HDL and sepsis hospital mortality (Fig. 2). The results of smooth curve and cox regression showed that there was a linear relationship between TG, HDL, TG/HDL level and hospital mortality of sepsis after adjustment according to fully adjusted model 4 . With the increase of TG and TG/HDL, the mortality of sepsis decreased gradually, and the levels of TG and TG/HDL were negatively correlated with hospital mortality of sepsis. It is worth mentioning that this phenomenon did not occur in $\mathrm{HDL}$, with the increase of $\mathrm{HDL}$, sepsis hospital mortality gradually increased. TG, $\mathrm{HDL}$, and TG/HDL were evenly divided into two groups according to the number of people, and K-M curves were drawn. In the higher TG, TG/HDL group, the septic patients had lower hospital mortality. Among the groups with higher levels of HDL, patients with sepsis had higher hospital mortality. (Fig. 2-7)

\subsection{Subgroup analysis}

In order to better explain this result, we carried out subgroup analysis and interaction test. In this study, groups were grouped according to age, sex, and presence of liver disease to verify whether the relationship between TG, HDL, AND TG/HDL and sepsis in-hospital mortality still held true in all subgroups. The results showed that there was no significant interaction between TG, TG/HDL level and hospital mortality in patients with sepsis in different age, sex and race. In addition, we found a strong association between HDL levels and sepsis in-hospital mortality in participants with liver disease, with HR and $95 \%$ confidence intervals of 14.08 (3.54 to 55.97 ). This strongly suggests that in patients with liver disease, the higher the HDL value, the higher the mortality rate. (Fig. 8-10)

\section{Discussion}

So far, the hospital mortality rate of sepsis remains high. Elucidating the risk factors or protective factors related to its mortality can provide key clues for improving its prognosis[21]. There is growing evidence that some laboratory indicators of blood lipids play a key role in the prognosis of sepsis. However, the relationship between TG, HDL and TG/HDL in the prognosis of sepsis is still not fully understood and further study is needed.

In this study, we first used univariate COX regression to analyze which factors might be associated with poor prognosis of sepsis, and then used multivariate COX regression to analyze the correlation between TG, HDL, TG/HDL and hospitalized mortality of sepsis. Univariate COX regression analysis showed that $\mathrm{HDL}$ was a protective factor for poor prognosis of sepsis, with an effect value and $95 \mathrm{Cl} \%$ confidence interval of $0.86(0.64,1.15), P=0.309$. Although $P$ value $>0.05$ was not considered as statistically significant, it was still clinically significant due to its significant effect value. This result is consistent with some previous studies in which patients with higher HDL levels had better sepsis outcomes than patients with lower HDL levels. An animal experiment conducted by Morin et al. showed that the mice with HDL deficiency were prone to infection and death, while the mice with increased HDL level due to increased ApolA1 expression had a lower mortality rate[18]. This was also well demonstrated in the study conducted by Dai et al.[22], in which recombinant HDL infusion reduced endotoxins, reduced 
inflammation, battled hypotensive responses, and improved survival in sepsis mice. This result theoretically supports the results of our univariate analysis. At the same time, our univariate analysis showed that TG and TG/HDL were not correlated with sepsis mortality, and their effect values (HR) and $95 \%$ confidence intervals $(\mathrm{Cl})$ were $0.95(0.87,1.03)$ and $0.98(0.95,1.02)$, respectively, with $P>0.05$ for both.

The results of our multivariate COX regression are not consistent with those of univariate COX regression. Under the premise of controlling the potential confounding factors, multivariate COX regression analysis of the relationship between TG, HDL, TG/HDL and poor prognosis of sepsis showed that TG and TG/HDL were negatively correlated with poor prognosis of sepsis, and their effect values HR and $95 \%$ confidence interval were $0.90(0.80-0.998), P=0.036$ and $0.95(0.91-0.99), P=0.049$, respectively. There was a positive correlation between HDL and poor prognosis of sepsis, and its effect value and $95 \%$ confidence interval were $1.14(0.8-1.63)$ and $\mathrm{P}=0.469$, respectively. Although the $\mathrm{P}>0.05$ showed no statistical significance, we still believed that HDL was positively correlated with poor prognosis of sepsis due to its significant clinical effect value. This is in stark contrast to previous findings that higher HDL levels are more likely to lead to an increased in-hospital sepsis mortality rate. In view of this, we conducted a survivability analysis of the results. We also found this in the survival analysis (Fig. 5-7). We divided TG, $\mathrm{HDL}$, and TG/HDL into two groups according to the number of people. The results showed that the mortality of patients in the group with higher HDL was higher, while that in the group with lower TG and TG/HDL was higher. A prospective, multicentre, randomized controlled phase II study by Dellinger RP et al was also disappointing: HDL infusion in patients with gram-negative bacteria-induced sepsis did not reduce the incidence of 28-day all-cause mortality or new organ failure, and the phase III trial was discontinued because of adverse reactions[23]. In 2020, Liu et al.[24] conducted a meta-analysis of the correlation between HDL and sepsis prognosis, which found that low HDL level was associated with higher mortality in adult patients with sepsis (OR and $95 \% \mathrm{Cl}$ were $2.00,1.23-3.24, \mathrm{P}=0.005$.). This conclusion supports the negative correlation between HDL levels and sepsis mixtures, but it is worth noting that the sample sizes of sepsis patients in this study were $11,28,30,51,65,68,108$, respectively[2531]. Compared with our samples, the average sample size is too small. Our sample size is relatively more representative.

In this study, we found that high levels of TG may be a protective factor in patients with sepsis. Survival analysis showed that patients with higher TG levels had a better prognosis. Luan et al. found that growth and differentiation factor 15(GDF15) is a factor essential for the survival of bacterial and viral infections and sepsis[32]. The protective effect of this factor is largely independent of the degree of pathogen control or inflammatory response, and the regulation of this factor requires the participation of TG. This explains the relationship between TG and sepsis prognosis to some extent. In this study, we found that the association between TG/HDL and poor prognosis of sepsis was not as significant as the association between single TG or HDL and poor prognosis of sepsis. This suggests that TG or HDL alone is better than TG/HDL ratios in predicting poor prognosis in patients with sepsis. 
In subgroup analysis, we found that TG and TG/HDL were stable in all subgroups. It is worth mentioning that in sepsis patients with liver disease, the association between HDL level and sepsis was strengthened, with an effect value and $95 \% \mathrm{Cl}$ of $14.08(3.54-55.97)$. $\mathrm{HDL}$ is known to be synthesized mainly in the liver and small intestine[33]. Maintaining high levels of HDL increases the burden on the liver. We hypothesize that in sepsis patients with liver disease, a high level of liver burden reduces immunity and ultimately leads to an increase in in-hospital mortality.

In this study, there are still some deficiencies: First, this study is a retrospective study, which inevitably has some selective bias. Secondly, the time span of this study is large, from 2008 to 2019. The detection method of TG or HDL may change during this period, but the detection method cannot be identified by the MIMIC-IV database at present. But given that the samples in the database are all from the same medical center, we think the effect is likely to be small. In this study, some of our work is also worthy of affirmation: compared with previous studies, our sample size was larger and more confounders were excluded. Compared with previous studies, our results better reflected the risk of TG, HDL and TG/HDL and sepsis hospitalization mortality. At the same time, we carried out multiple interpolation for the missing data to maximize the statistical capability and minimize the error. We also performed more subgroup analyses to make our results more robust.

\section{Conclusion}

To sum up, we clarified that there was a negative correlation between TG, TG/HDL and the poor prognosis of sepsis, while HDL was positively correlated with the poor prognosis of sepsis. Our results also suggest that the correlation between HDL and poor prognosis of sepsis is enhanced in patients with sepsis with liver disease. However, these results still need to be verified by more basic trials and largescale clinical trials.

\section{Abbreviations}

Abbreviations: SD = Standard Deviation; LOS = Length of Stay; SOFA = Sequential Organ Failure Assessment; SBP = Systolic Blood Pressure; DBP = Diastolic Blood Pressure; MBP = Mean Blood Pressure; $\mathrm{WBC}=$ White Blood Cell; IQR = Interquartile Range; $A L B=$ Albumin; BUN = Blood Urea Nitrogen; CRE = Creatinine; $\mathrm{ALT}=$ Alanine aminotransferase; $\mathrm{AST}=$ Aspartate aminotransferase; $\mathrm{TBIL}=$ Total Bilirubin; $\mathrm{TG}$ = Triglycerides; $\mathrm{HDL}=$ High-Density Lipoprotein.

\section{Declarations}

\section{Compliance with Ethical Standards}

\section{Authors' contributions}

G and $W$ conceived the idea; $G, T$ and $D$ wrote the manuscript; $X, H$ and $S$ collected and read the literature and revised the article; $W$ and $G$ read through and corrected the manuscript. All authors read and 
approved the final manuscript. $\mathrm{G}$ is the first author. Tian is the co-first author. $\mathrm{W}$ and $\mathrm{G}$ are the corresponding author of this paper.

\section{Competing interests}

The authors declare that they have no competing interests.

\section{Availability of data and materials}

Data can be downloaded from 'MIMIC-IV' database (https://physionet.org/content/mimiciv/0.4/).

\section{Consent for publication}

Not applicable

\section{Ethics approval and consent to participate}

Not applicable

\section{Funding}

This work was Supported by Grants from National Natural Science Foundation of China (81860370), General Project of Natural Science Foundation of Qinghai Province (2019-ZJ-970Q), CAS "Light of West China" Program (2019) and Qinghai Scientific Innovation Project of Traditional Chinese-Tibetan Medicine (J2020007).

\section{Acknowledgements}

Thank Tianyang Hu for his help in data processing, Xiaoxing Wei for his guidance, and all the authors for their valuable discussion

\section{References}

1. Singer M, et al., The Third International Consensus Definitions for Sepsis and Septic Shock (Sepsis-3). Jama, 2016. 315(8): p. $801-10$.

2. Rudd KE, et al. Global, regional, and national sepsis incidence and mortality, 1990-2017: analysis for the Global Burden of Disease Study. Lancet. 2020;395(10219):200-11.

3. Fleischmann-Struzek $C$, et al. Incidence and mortality of hospital- and ICU-treated sepsis: results from an updated and expanded systematic review and meta-analysis. Intensive Care Med. 2020;46(8):1552-62.

4. Iwashyna TJ, et al. Population burden of long-term survivorship after severe sepsis in older Americans. J Am Geriatr Soc. 2012;60(6):1070-7. 
5. Markwart R, et al. Epidemiology and burden of sepsis acquired in hospitals and intensive care units: a systematic review and meta-analysis. Intensive Care Med. 2020;46(8):1536-51.

6. Chait A, et al. Remnants of the Triglyceride-Rich Lipoproteins, Diabetes, and Cardiovascular Disease. Diabetes. 2020;69(4):508-16.

7. Chapman MJ, et al. Triglyceride-rich lipoproteins and high-density lipoprotein cholesterol in patients at high risk of cardiovascular disease: evidence and guidance for management. Eur Heart $\mathrm{J}$. 2011;32(11):1345-61.

8. Rader DJ, Hovingh GK. HDL and cardiovascular disease. Lancet. 2014;384(9943):618-25.

9. Rysz J, et al., The Role and Function of HDL in Patients with Chronic Kidney Disease and the Risk of Cardiovascular Disease. Int J Mol Sci, 2020. 21(2).

10. Mahdy Ali K, et al. Cardiovascular disease risk reduction by raising HDL cholesterol-current therapies and future opportunities. Br J Pharmacol. 2012;167(6):1177-94.

11. Vaziri ND. HDL abnormalities in nephrotic syndrome and chronic kidney disease. Nat Rev Nephrol. 2016;12(1):37-47.

12. Vaziri ND, Navab M, Fogelman AM. HDL metabolism and activity in chronic kidney disease. Nat Rev Nephrol. 2010;6(5):287-96.

13. Young KA, et al. The triglyceride to high-density lipoprotein cholesterol (TG/HDL-C) ratio as a predictor of insulin resistance, $\beta$-cell function, and diabetes in Hispanics and African Americans. $J$ Diabetes Complications. 2019;33(2):118-22.

14. Weiler Miralles CS, et al. Waist-to-height ratio (WHtR) and triglyceride to HDL-C ratio (TG/HDL-c) as predictors of cardiometabolic risk. Nutr Hosp. 2015;31(5):2115-21.

15. Xia W, et al. Elevated TG/HDL-C and non-HDL-C/HDL-C ratios predict mortality in peritoneal dialysis patients. BMC Nephrol. 2020;21(1):324.

16. Olabode $\mathrm{OP}$, et al. Triglyceride/HDL-cholesterol ratio and plasminogen activator inhibitor-1 independently predict high pulse pressure in sickle cell trait and disease. Arch Physiol Biochem. 2020;126(2):166-71.

17. Gong R, et al., Associations of TG/HDL Ratio with the Risk of Prediabetes and Diabetes in Chinese Adults: A Chinese Population Cohort Study Based on Open Data. Int J Endocrinol, 2021. 2021: p. 9949579.

18. Morin EE, et al. HDL in sepsis - risk factor and therapeutic approach. Front Pharmacol. 2015;6:244.

19. Johnson AE, et al. MIMIC-III, a freely accessible critical care database. Sci Data. 2016;3:160035.

20. Gong R, Xu Z, Wei X. The association between vitamin D3 and diabetes in both hyperuricemia and non-hyperuricemia populations. Endocrine, 2021.

21. Salomão R, et al. Sepsis: evolving concepts and challenges. Braz J Med Biol Res. 2019;52(4):e8595.

22. Dai $L$, et al. The apolipoprotein A-I mimetic peptide $4 \mathrm{~F}$ prevents defects in vascular function in endotoxemic rats. J Lipid Res. 2010;51(9):2695-705. 
23. Dellinger RP, et al. Efficacy and safety of a phospholipid emulsion (GR270773) in Gram-negative severe sepsis: results of a phase II multicenter, randomized, placebo-controlled, dose-finding clinical trial. Crit Care Med. 2009;37(11):2929-38.

24. Liu SH, et al. Effect of low high-density lipoprotein levels on mortality of septic patients: A systematic review and meta-analysis of cohort studies. World J Emerg Med. 2020;11(2):109-16.

25. Shor R, et al. Low HDL levels and the risk of death, sepsis and malignancy. Clin Res Cardiol. 2008;97(4):227-33.

26. Roveran Genga K, et al. Two-year follow-up of patients with septic shock presenting with low HDL: the effect upon acute kidney injury, death and estimated glomerular filtration rate. J Intern Med. 2017;281(5):518-29.

27. Chien YF, et al. Decreased serum level of lipoprotein cholesterol is a poor prognostic factor for patients with severe community-acquired pneumonia that required intensive care unit admission. $J$ Crit Care. 2015;30(3):506-10.

28. Lekkou A, et al. Serum lipid profile, cytokine production, and clinical outcome in patients with severe sepsis. J Crit Care. 2014;29(5):723-7.

29. Grion CM, et al. Lipoproteins and CETP levels as risk factors for severe sepsis in hospitalized patients. Eur J Clin Invest. 2010;40(4):330-8.

30. Cirstea $\mathrm{M}$, et al. Decreased high-density lipoprotein cholesterol level is an early prognostic marker for organ dysfunction and death in patients with suspected sepsis. J Crit Care. 2017;38:289-94.

31. Lee SH, et al., Prognostic Implications of Serum Lipid Metabolism over Time during Sepsis. Biomed Res Int, 2015. 2015: p. 789298.

32. Luan HH, et al. GDF15 Is an Inflammation-Induced Central Mediator of Tissue Tolerance. Cell. 2019;178(5):1231-44.e11.

33. Constantinou $\mathrm{C}$, et al. Advances in high-density lipoprotein physiology: surprises, overturns, and promises. Am J Physiol Endocrinol Metab. 2016;310(1):E1-14.

\section{Figures}




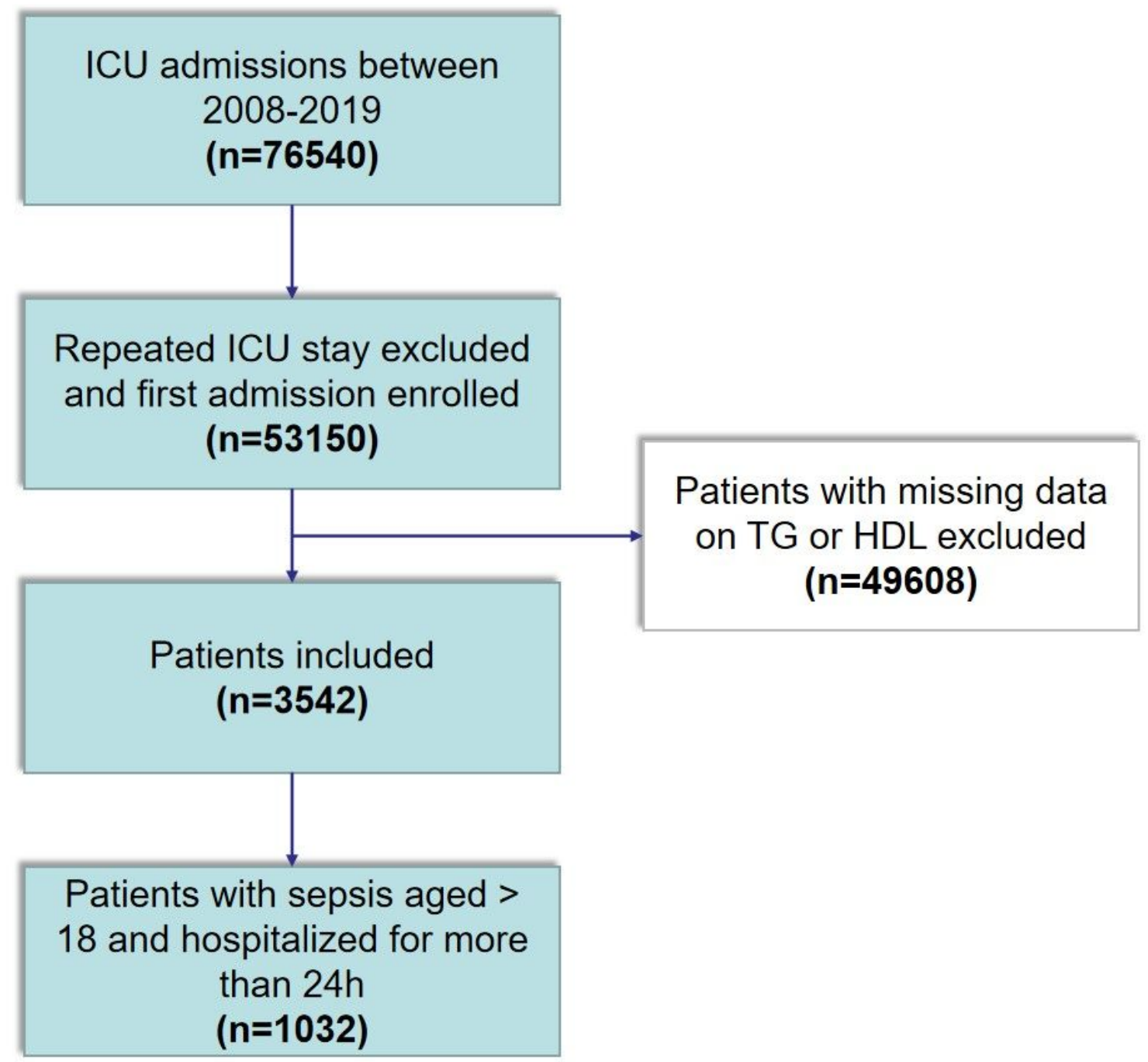

Figure 1

Flowchart of patient selection in this study. 


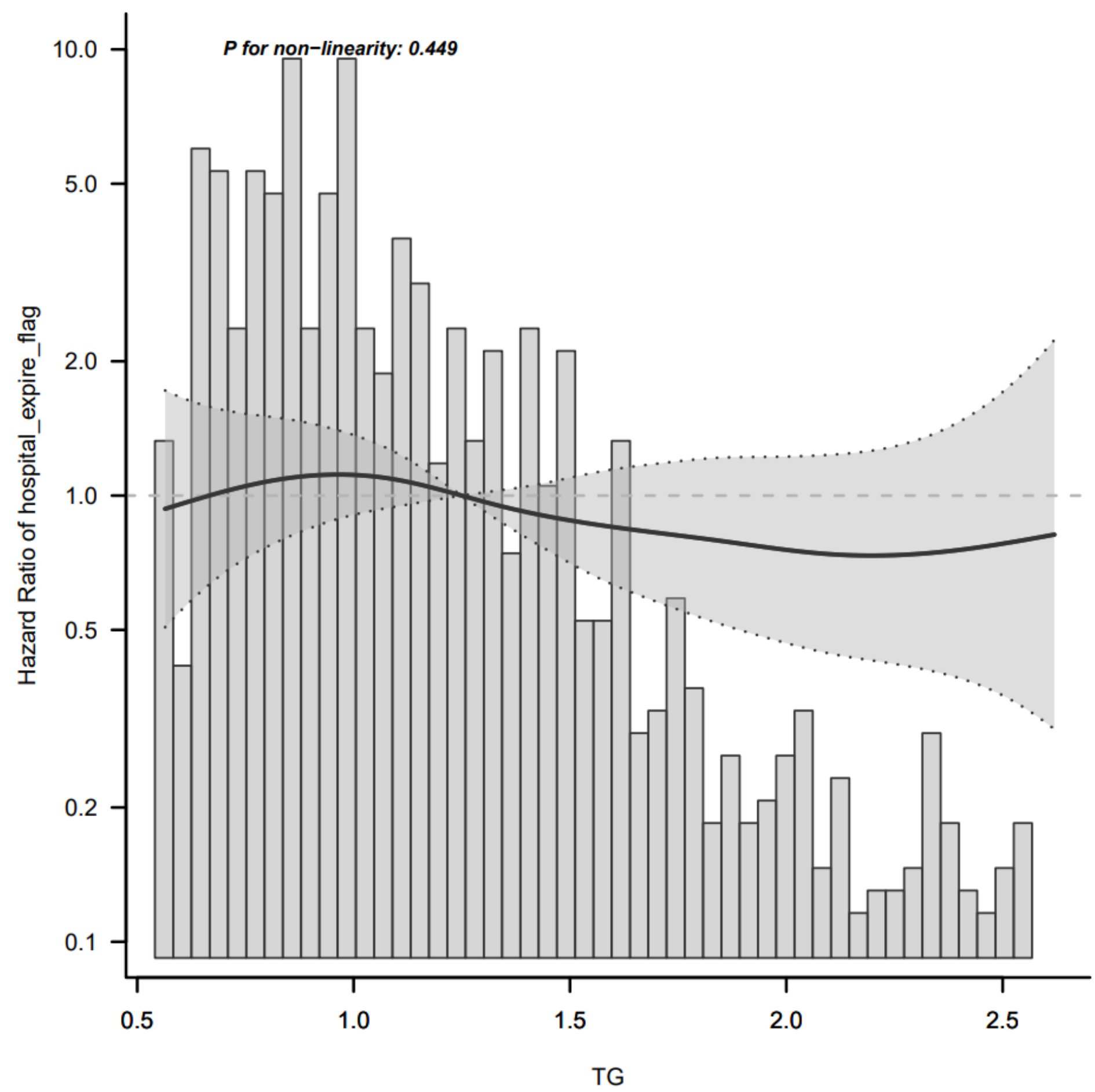

Figure 2

Association between TG and Sepsis in-hospital mortality. 


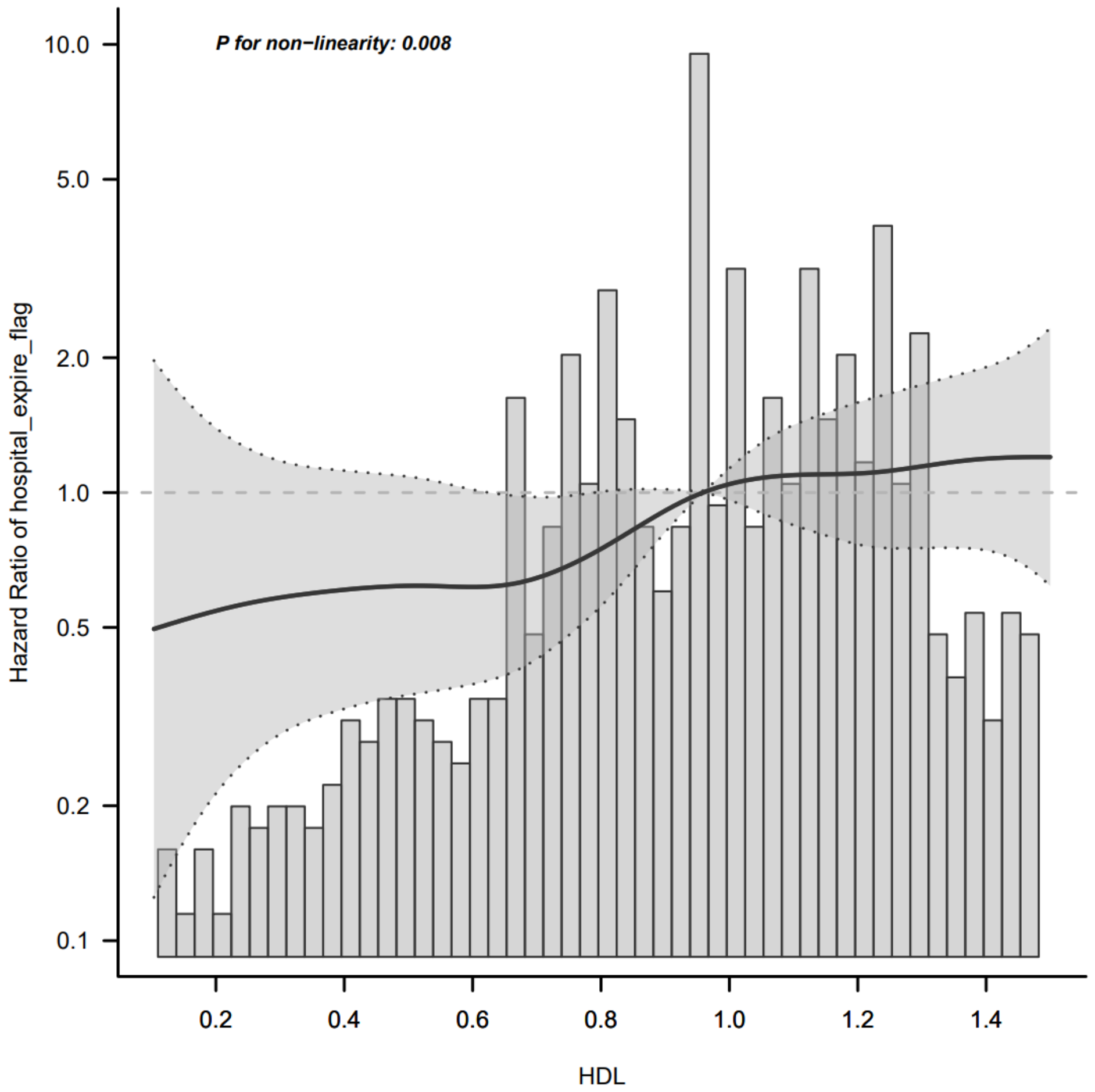

Figure 3

Association between HDL and Sepsis in-hospital mortality. 


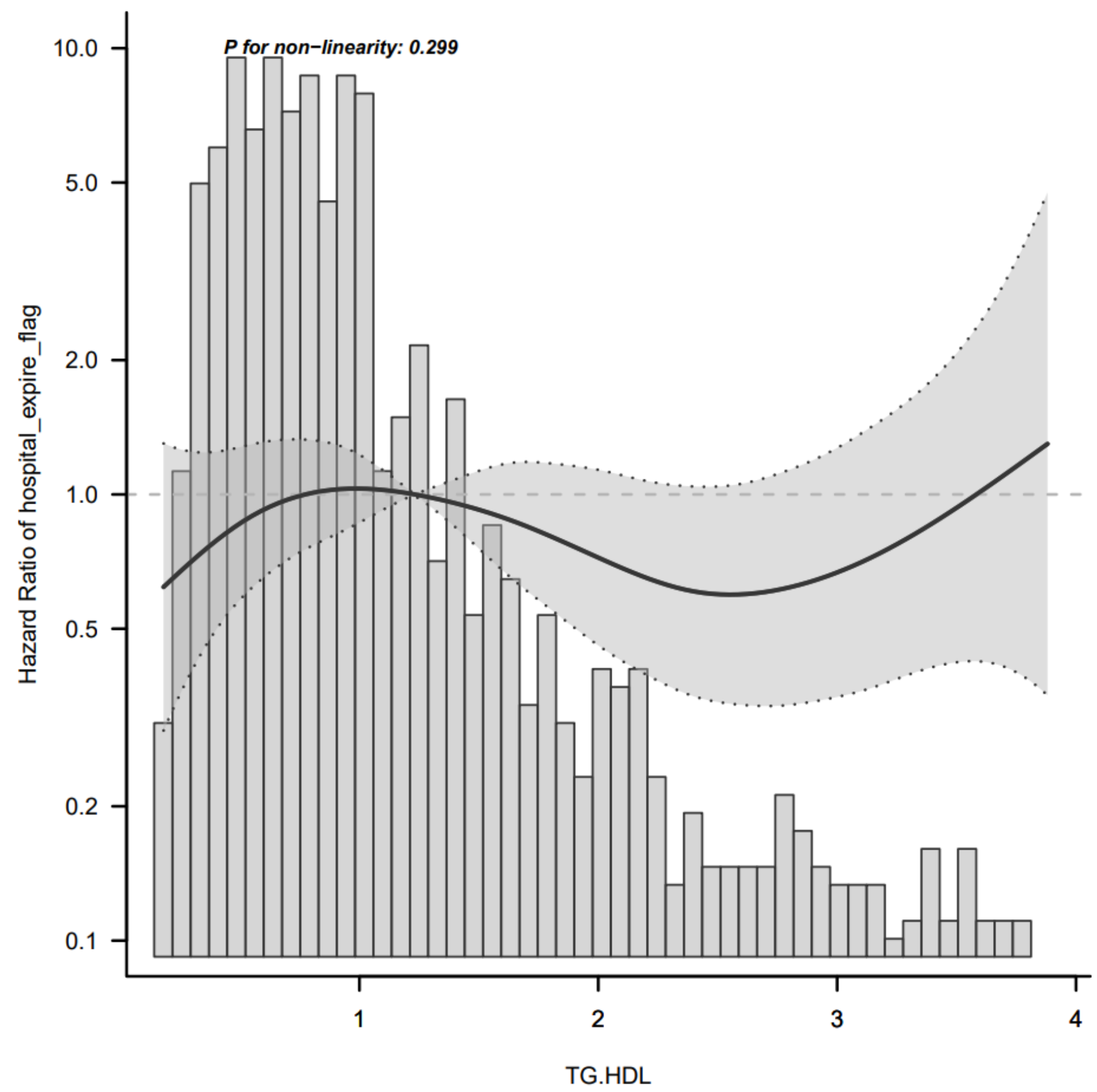

Figure 4

Association between TG/HDL and Sepsis in-hospital mortality. 


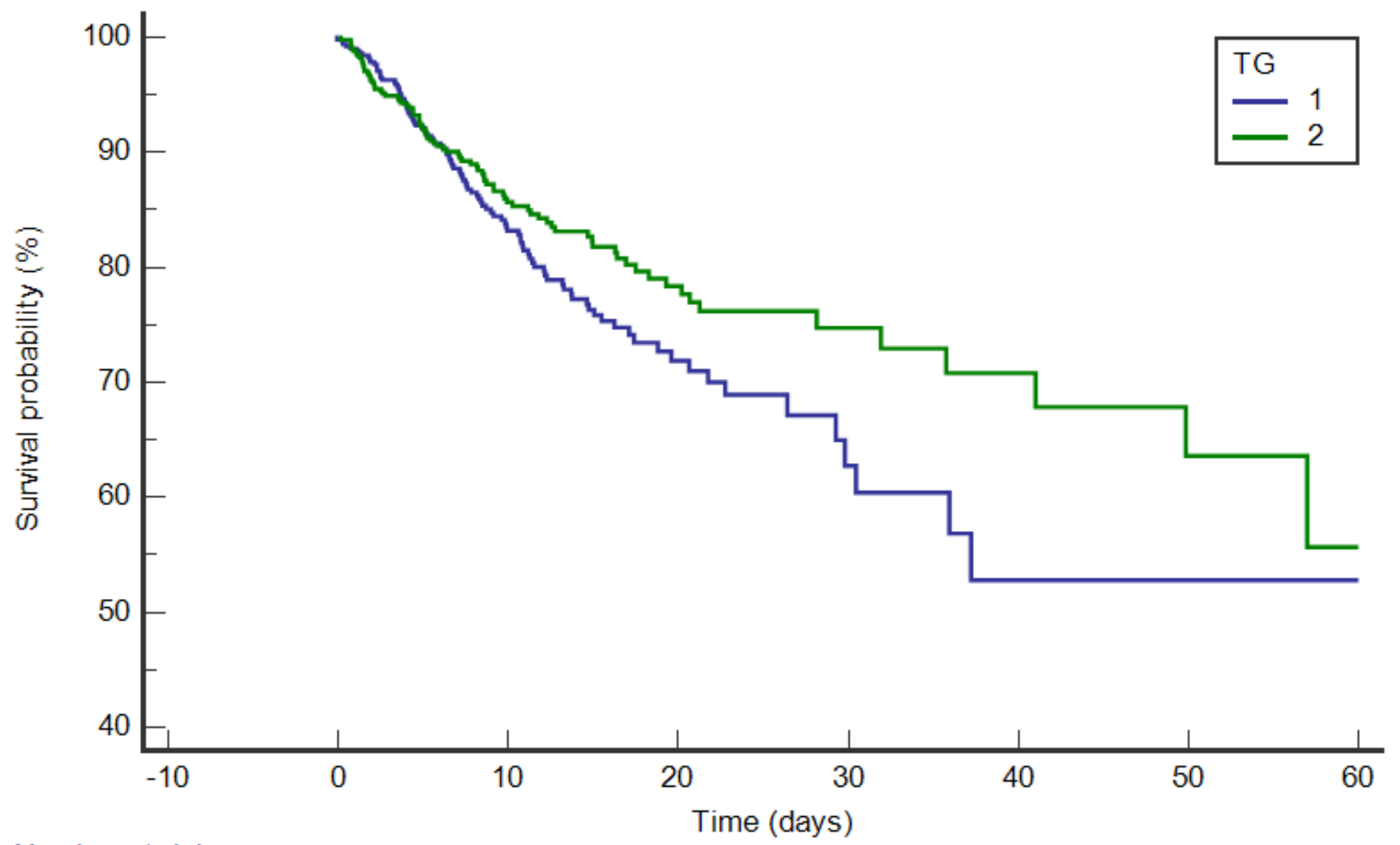

Number at risk

\begin{tabular}{|c|c|c|c|c|c|c|c|}
\hline \\
\hline 517 & 516 & 255 & 85 & 27 & 11 & 5 & 1 \\
\hline Group: 2 & & & & & & & \\
\hline 515 & 515 & 261 & 114 & 46 & 24 & 14 & 6 \\
\hline
\end{tabular}

Figure 5

Survival analysis of Sepsis in-hospital mortality with TG. 


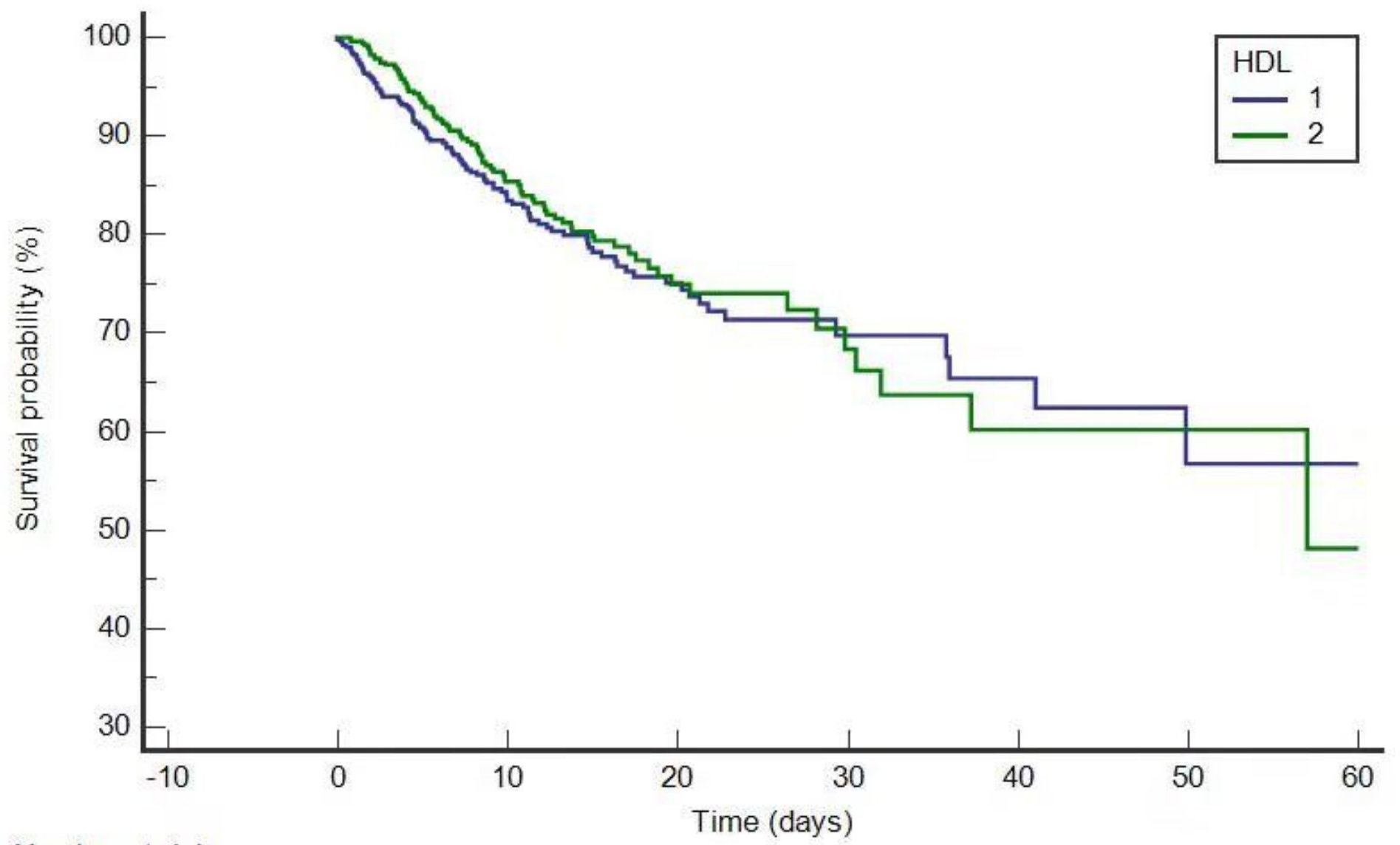

Number at risk

Group: 1

Group: 2

511 520 269 113 41

22

10

3

511

247

86

32

13

9

4

Figure 6

Survival analysis of Sepsis in-hospital mortality HDL. 


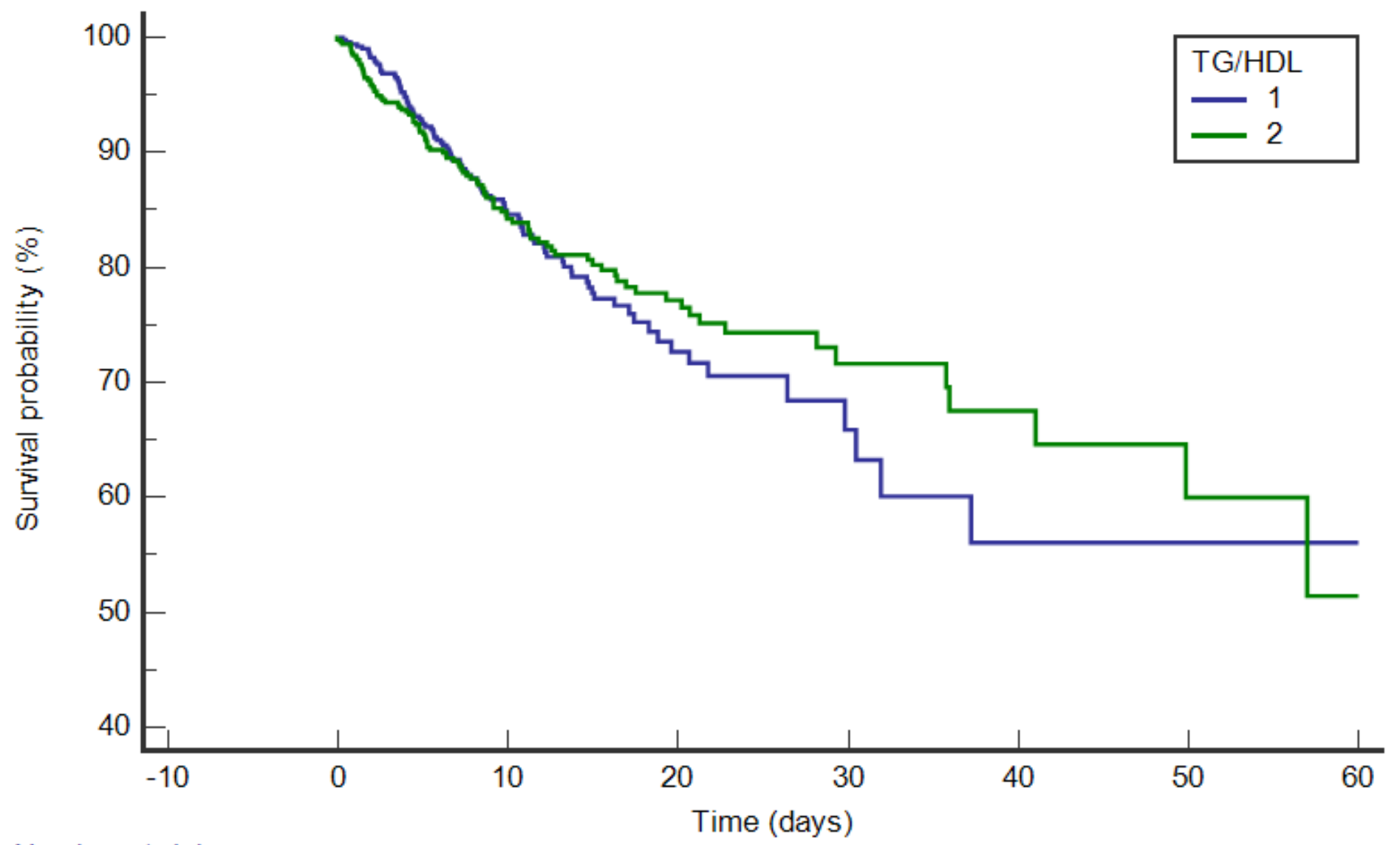

Number at risk

\begin{tabular}{|c|c|c|c|c|c|c|c|}
\hline Group: 1 & & & & & & & \\
\hline 516 & 516 & 250 & 78 & 26 & 12 & 7 & 2 \\
\hline Group: 2 & & & & & & & \\
\hline 516 & 515 & 266 & 121 & 47 & 23 & 12 & 5 \\
\hline
\end{tabular}

Figure 7

Survival analysis of Sepsis in-hospital mortality with TG/HDL ratio. 


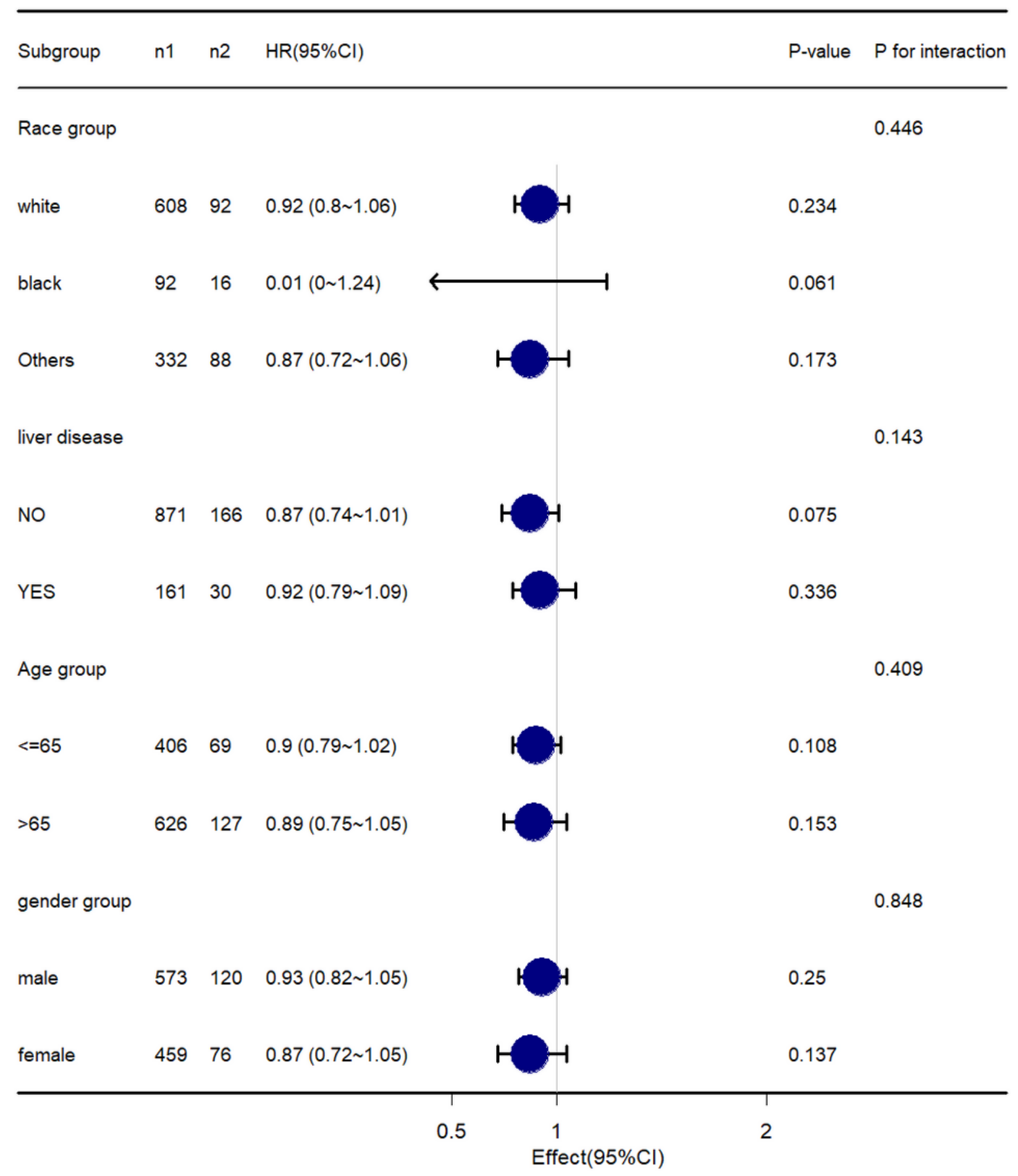

\section{Figure 8}

Subgroup analyses of the association between TG and Sepsis in-hospital mortality. 


\begin{tabular}{|c|c|c|c|c|c|}
\hline Subgroup & $\mathrm{n} 1$ & $\mathrm{n} 2$ & $\mathrm{HR}(95 \% \mathrm{Cl})$ & $P$-value & $P$ for interaction \\
\hline Race group & & & & & 0.12 \\
\hline white & 608 & 92 & $0.82(0.47 \sim 1.45)$ & 0.5 & \\
\hline black & 92 & 16 & $1.87(0.07 \sim 51.61)$ & 0.712 & \\
\hline Others & 332 & 88 & $1.94(1.13 \sim 3.34)$ & 0.016 & \\
\hline liver disease & & & & & 0.003 \\
\hline NO & 871 & 166 & $0.88(0.59 \sim 1.3)$ & 0.514 & \\
\hline YES & 161 & 30 & $14.08(3.54 \sim 55.97)$ & $<0.001$ & \\
\hline Age group & & & & & 0.045 \\
\hline$<=65$ & 406 & 69 & $2.88(1.54 \sim 5.36)$ & 0.001 & \\
\hline$>65$ & 626 & 127 & $0.73(0.46 \sim 1.16)$ & 0.179 & \\
\hline gender group & & & & & 0.094 \\
\hline male & 573 & 120 & $1.2(0.75 \sim 1.91)$ & 0.445 & \\
\hline female & 459 & 76 & $1.1(0.59 \sim 2.05)$ & 0.767 & \\
\hline
\end{tabular}

\section{Figure 9}

Subgroup analyses of the association between HDL and Sepsis in-hospital mortality. 


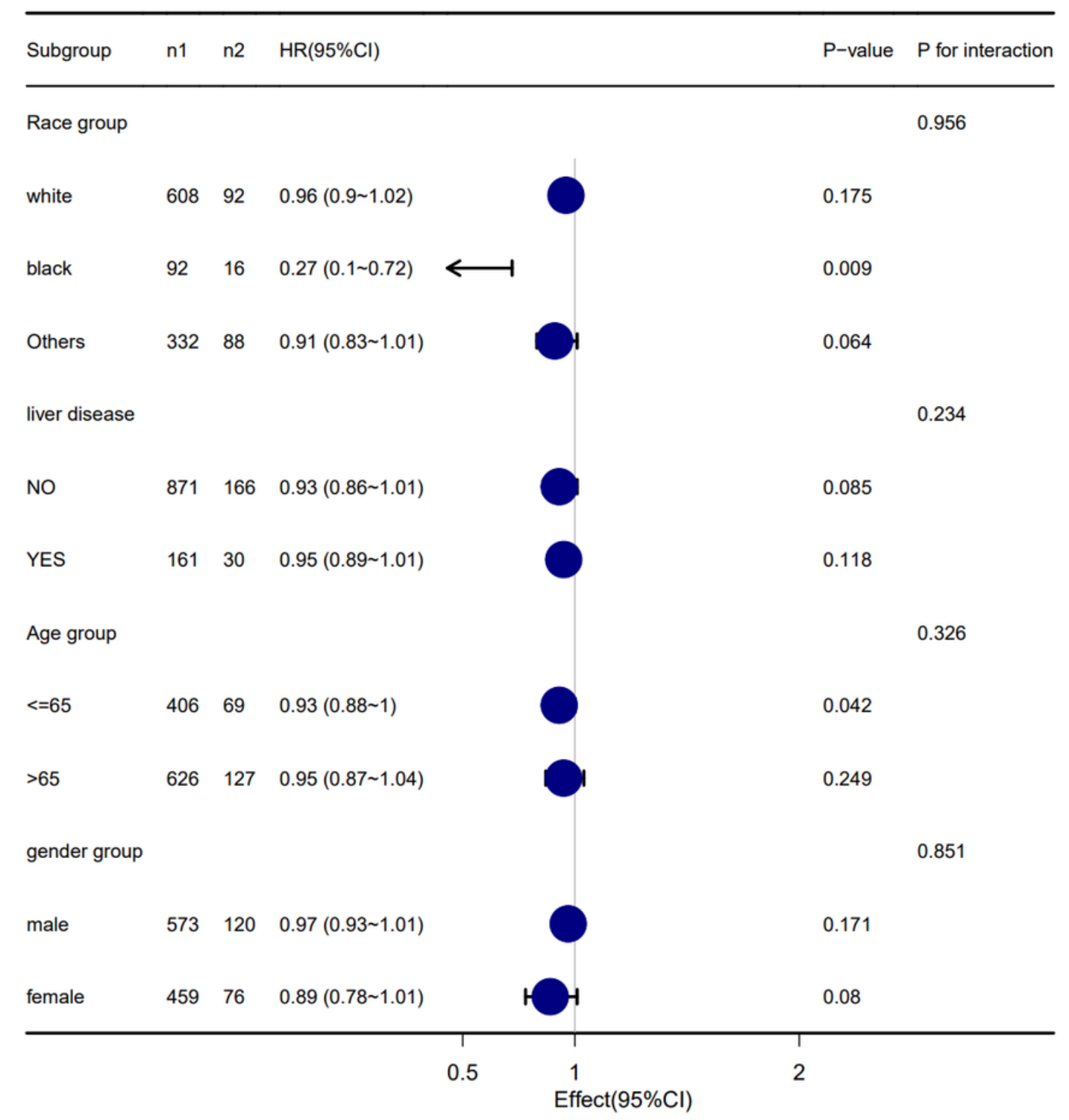

\section{Figure 10}

Subgroup analyses of the association between TG/HDL and Sepsis in-hospital mortality. 